Pacific

Journal of

Mathematics

THE ENDING LAMINATION CONJECTURE FOR HYPERBOLIC THREE-MANIFOLDS WITH SLENDER END-INVARIANTS

Richard Allen Evans 


\title{
THE ENDING LAMINATION CONJECTURE FOR HYPERBOLIC THREE-MANIFOLDS WITH SLENDER END-INVARIANTS
}

\author{
RiCHARD ALLEN EVANS
}

\begin{abstract}
Using a density theorem and a drilling theorem of Bromberg we prove a uniqueness result for singly degenerate hyperbolic 3-manifolds without cusps. By results of Minsky on the curve complex and end-invariants we then improve upon this theorem to prove the ending lamination conjecture for singly degenerate hyperbolic 3-manifolds with slender end-invariants. Although this result is known by work of Brock, Canary and Minsky, our proof uses a different approach, in particular avoiding the construction of a model manifold.
\end{abstract}

\section{Introduction}

An orientable hyperbolic 3-manifold is a quotient $\mathbb{H}^{3} / \Gamma$, where $\Gamma$ is a discrete subgroup of $\mathrm{PSL}_{2}(\mathbb{C})$. Under the natural identification of $\mathrm{PSL}_{2}(\mathbb{C})$ with the group Isom $_{+} \mathbb{H}^{3}$ of orientation preserving isometries of $\mathbb{H}^{3}$, we can equivalently consider $\Gamma$ as a subgroup of Isom $_{+} \mathbb{H}^{3}$ acting properly discontinuously on $\mathbb{\boxplus}^{3}$.

One of the fundamental problems in the study of hyperbolic 3-manifolds is to provide conditions that determine the manifold uniquely. Thurston's ending lamination conjecture proposes that a geometrically tame hyperbolic 3-manifold is uniquely determined by its topological type and a collection of end-invariants. An end-invariant is either a conformal structure on a surface or a measured lamination on a surface.

In the case of finite volume hyperbolic 3-manifolds the conjecture is true by the classical Mostow-Prasad rigidity theorem. For geometrically finite infinite-volume hyperbolic 3-manifolds (equivalently manifolds for which all end-invariants are conformal structures) the conjecture is true by work of Ahlfors, Bers, Kra, Marden, Maskit, Sullivan, Thurston et al. The earliest results involving geometrically infinite hyperbolic 3-manifolds were obtained by Minsky in two distinct settings. The first, in [Minsky 1994], is for hyperbolic 3-manifolds with compact cores in which every boundary component is incompressible and such that there is a positive

MSC2000: primary 57M50; secondary 30F40, 57N10.

Keywords: hyperbolic 3-manifolds, Kleinian groups, ending lamination conjecture. 
lower bound to the injectivity radius. The second, in [Minsky 1999], is a general result in the case that the hyperbolic 3-manifold has pared relative compact core $(F \times I, \partial F \times I)$, where $\operatorname{int}(F)$ is homeomorphic to a once-punctured torus. More recently, Brock, Canary and Minsky [Brock et al. 2004] have proved the conjecture in full in the case that the compact core has incompressible boundary. One of the key steps in both of Minsky's papers and in the Brock-Canary-Minsky proof is the construction of a model manifold together with a bilipschitz map from this model to the hyperbolic 3-manifold given the topological type and the end-invariants of the hyperbolic 3-manifold. It is this step that we are able to avoid in this paper.

In contrast to [Minsky 1994], we will consider hyperbolic 3-manifolds with unbounded geometry. This means that their injectivity radius is not bounded away from zero outside of the cusp components. Equivalently there is no positive lower bound to the length of closed geodesics in the manifold.

We will further restrict the class of manifolds within those of unbounded geometry. Let $S$ be a compact orientable surface. A pair-of-pants multicurve is a collection of disjoint simple closed curves on $S$ that give a pair-of-pants decomposition of $S$. In a hyperbolic 3-manifold a geodesic pair-of-pants multicurve is a collection of geodesics that have a pair-of-pants multicurve representative on some component of the boundary of a relative compact core minus the parabolic locus. The length $\ell(\alpha)$ of a geodesic pair-of-pants multicurve $\alpha$ is the sum of the lengths of the closed geodesic components.

Our first theorem is a uniqueness result for singly degenerate hyperbolic 3manifolds based upon the work of Bromberg [2002; 2004]:

Theorem 3.1. Suppose $N_{1}$ and $N_{2}$ are singly degenerate hyperbolic 3-manifolds without cusps with the same conformal boundary end-invariant and the same sequence of geodesic pair-of-pants multicurves with length going to zero. Then $N_{1}$ is isometric to $\mathrm{N}_{2}$.

For simplicity in this paper we have restricted attention to singly degenerate manifolds. With current technology the theorem can be immediately extended to hyperbolic 3-manifolds with pared incompressible compact cores in which corresponding geometrically infinite ends of each manifold have the same unbounded length exiting sequence of geodesic pair-of-pants multicurves. In future work we will generalise these results to the class of hyperbolic 3-manifolds with unbounded geometry.

Beautiful new developments by Minsky [2000; 2001; 2003], using work of Masur and Minsky [1999; 2000], allow one to determine which geodesics associated to an (incompressible) geometrically infinite end are short based solely upon the combinatorics of curves in the curve complex associated to the measured lamination end-invariant of this end. Using these estimates Minsky [2003] was able 
to construct a model manifold and a lipschitz map from this model manifold to a hyperbolic 3-manifold where the model manifold depends upon the topological type of the hyperbolic 3-manifold and its end-invariants. The already cited article [Brock et al. 2004] promotes this lipschitz map to a bilipschitz map, proving the ending lamination conjecture.

Using the results of Minsky we are able to prove the ending lamination conjecture for singly degenerate hyperbolic 3-manifolds without cusps with slender ending lamination end-invariants (see Theorem 3.2).

Theorem 1.2. If $N$ is a singly degenerate hyperbolic 3-manifold without cusps with a slender ending lamination end-invariant then it is uniquely determined by its end-invariants.

Since it is not immediately clear that there are any manifolds with slender endinvariants, in Section 4 we construct examples of such manifolds. We note that this is a construction given by Minsky in [Minsky 2000] and relies upon the results contained within that paper.

\section{Preliminaries}

Let $N$ be a hyperbolic 3-manifold. The convex core, $\mathscr{C}(N)$, of $N$ is the smallest convex submanifold whose inclusion is a homotopy equivalence.

Let $\Gamma=\pi_{1}(N)$. Then $\Gamma$ acts a group of conformal automorphisms of the boundary at infinity of $\mathbb{H}^{3}$ which we will identify with $\overline{\mathbb{C}}$. The domain of discontinuity for the action of $\Gamma$ on $\overline{\mathbb{C}}$ is denoted $\Omega_{\Gamma}$ and is the largest open subset upon which $\Gamma$ acts properly discontinuously. The complement is the limit set, $\Lambda_{\Gamma}$. For a singly degenerate hyperbolic 3-manifold the associated domain of discontinuity is connected and simply connected.

Given a hyperbolic 3-manifold $N$ the associated Kleinian manifold is $\hat{N}=\left(\mathbb{H}^{3} \cup\right.$ $\left.\Omega\left(\pi_{1}(N)\right)\right) / \pi_{1}(N)$. If $N$ is a singly degenerate hyperbolic 3-manifold without cusps it is homeomorphic to $S \times(-\infty, \infty)(S$ a closed surface of genus at least 2) by [Bonahon 1986] and the associated Kleinian manifold $\hat{N}$ is homeomorphic to $S \times[0, \infty)$.

The injectivity radius of a point $x \in N, i n j_{N}(x)$, is half the length of the shortest homotopically nontrivial loop passing through $x$. For $\varepsilon>0$ we define the $\varepsilon$-thin part of $N$ to be $N_{\operatorname{thin}(\varepsilon)}=\left\{x \in N \mid i \operatorname{inj}_{N}(x)<\varepsilon\right\}$, and the $\varepsilon$-thick part of $N$ to be $N_{\text {thick }(\varepsilon)}=N-N_{\operatorname{thin}(\varepsilon)}$. A result of Margulis gives a universal constant (the Margulis constant) $M_{3}$ such that if $\varepsilon<M_{3}$ then a component of $N_{\text {thin( }(\varepsilon)}$ is a solid cusp cylinder, a torus cusp or a solid torus neighbourhood of a closed geodesic. A component of the first (respectively second) type is a rank-1 (respectively rank-2) cusp. The collection of components of the first and second type are the $\varepsilon$-thin cusp components of $N$ or simply the cusp components of $N$. 
A hyperbolic 3-manifold is geometrically finite if the convex core minus its intersection with all cusp components is compact. Otherwise the manifold is geometrically infinite.

We note that the only thin components of a singly degenerate hyperbolic 3manifold without cusps are solid torus neighbourhoods of closed geodesics.

Deformation spaces and Bers slices. (For a discussion of deformation spaces of hyperbolic 3-manifolds see for instance [Anderson 1998].) Fix a compact threemanifold $M$. The deformation space, $\mathscr{D}(M)$, associated to $M$ is the collection of hyperbolic 3-manifolds homotopy-equivalent to $M$ each with a fixed marking, that is, a homotopy equivalence from $M$ to the manifold. Two hyperbolic 3-manifolds are considered equivalent as elements of $\mathscr{D}(M)$ if there is an isometry between them in the homotopy class of the map between them determined by their markings.

An equivalent space is the space of all faithful representations

$$
\rho: \pi_{1}(M) \rightarrow \mathrm{PSL}_{2}(\mathbb{C})=\operatorname{Isom}_{+}\left(\mathbb{M}^{3}\right)
$$

with discrete image. Two representations are equivalent if there is an element of $\mathrm{PSL}_{2}(\mathbb{C})$ conjugating one to the other. We topologise this space with the algebraic topology by fixing some finite generating set $H$ of $\pi_{1}(M)$ and stating that representations $\left\{\rho_{i}\right\}$ converge to $\rho$ if $\rho_{i}(g)$ converges to $\rho(g)$ in $\operatorname{PSL}_{2}(\mathbb{C})$ for each $g \in H$. This topology is independent of the choice of $H$. A fundamental fact is that the deformation space $\mathscr{D}(M)$ is a closed subspace of the space of all representations of $\pi_{1}(M)$ into $\mathrm{PSL}_{2}(\mathbb{C})$ equipped with the same topology.

We now restrict attention to the case of interest in this paper. Let $S$ be a closed surface and let $M=S \times[0,1]$. Since $M$ is homotopy equivalent to $S$ the deformation space associated to $M$ is often denoted $\mathscr{D}(S)$. The interior of $\mathscr{D}(S)$ (in the full representation space) consists of geometrically finite manifolds without cusps. Equivalently it consists of those manifolds with a compact convex core. These are the quasifuchsian manifolds. If $N$ is a quasifuchsian manifold then the associated Kleinian manifold $\hat{N}$ is homeomorphic to $M=S \times[0,1]$.

By work of Ahlfors and Bers quasifuchsian manifolds are uniquely determined by their conformal boundary consisting of a pair of conformal structures on the surface $S$. Further, given a pair of conformal structures there is a quasifuchsian manifold in $\mathscr{D}(S)$ with this pair of structures as its conformal boundary. We will denote each manifold in the interior of $\mathscr{D}(S)$ by $Q F(X, Y)$, where $X$ and $Y$ are conformal structures on $S$.

A Bers slice is a subset of the interior of $\mathscr{D}(S)$ consisting of all quasifuchsian manifolds in which one of the conformal structures is fixed. If the fixed structure is $X$ we denote the Bers slice by $B_{X}$. A result of Bers is that each Bers slice has compact closure in $\mathscr{D}(S)$. 
Ends and end-invariants. (For a discussion of ends of hyperbolic three-manifolds see, for instance, [Canary 1993]. For a discussion of end-invariants see [Minsky 2001], for instance.)

An end of a hyperbolic 3-manifold is geometrically finite if it has a neighbourhood disjoint from the convex core. It is geometrically infinite otherwise.

Let $N$ be a singly degenerate hyperbolic 3-manifold without cusps. By [Bonahon 1986] $N$ is homeomorphic to $S \times(-\infty, \infty)$ where $S$ is a closed orientable surface of genus at least 2 and $N$ has two ends, one geometrically finite and the other geometrically infinite. Each end has a product neighbourhood homeomorphic to $S \times[0, \infty)$.

The end-invariant associated to each geometrically finite end of a hyperbolic 3-manifold is a conformal boundary component. The conformal boundary component, $X$, associated to the geometrically finite end of $N$ is the full conformal boundary: $X=\Omega_{\pi_{1}(N)} / \pi_{1}(N)$. Note that $X$ is homeomorphic to $S$.

The end-invariant associated to a geometrically infinite end of a hyperbolic 3manifold is a lamination. We describe one way to identify the lamination $\lambda$ for the manifold $N$ using the conformal boundary $X$. Bonahon's result [1986] implies that there is a sequence $\left\{c_{i}\right\}$ of simple closed curves on $X$, considered as the boundary component of the Kleinian manifold $\hat{N}$, such that the geodesic representatives $\left\{c_{i}^{*}\right\}$ of $\left\{c_{i}\right\}$ within $N$ exit the geometrically infinite end; that is, given any neighbourhood $U$ of the geometrically infinite end, all but finitely many of the geodesics are contained in $U$. Bonahon [1986] and Thurston [1979] show that the support of any limit measured geodesic lamination of a convergent subsequence of $\left\{c_{i}^{*} / \ell\left(c_{i}^{*}\right)\right\}$ (in the space of measured geodesic laminations on $X$ ) is unique. This support is the ending lamination $\lambda$.

Let $N$ and $N^{\prime}$ be two singly degenerate hyperbolic 3-manifolds without cusps in $\mathscr{D}(S)$ with associated end-invariants $(X, \lambda)$ and $\left(X^{\prime}, \lambda^{\prime}\right)$. Note that $X=\partial \hat{N}$ and $X^{\prime}=\partial \hat{N}^{\prime}$ where $\hat{N}$ and $\hat{N}^{\prime}$ are the associated Kleinian manifolds. Suppose $f: S \rightarrow N$ and $f^{\prime}: S \rightarrow N^{\prime}$ are the attached homotopy equivalences. The endinvariants of $N$ and $N^{\prime}$ are the same if

- there exists a homeomorphism $h: \hat{N} \rightarrow \hat{N}^{\prime}$ such that $\left.h\right|_{N}: N \rightarrow N^{\prime}$ is homotopic to the map $f^{\prime} \circ f^{-1}: N \rightarrow N^{\prime}$,

- there is a conformal map $X \rightarrow X^{\prime}$ in the homotopy class of $\left.h\right|_{X}: X \rightarrow X^{\prime}$, and - $\left.h\right|_{X}(\lambda)$ is homotopic to $\lambda^{\prime}$ in $X^{\prime}$.

Remark. As noted in the introduction, a hyperbolic 3-manifold with finitely generated fundamental group is uniquely determined by its end-invariants if each end-invariant is a conformal boundary component (equivalently if every end is geometrically finite). 
The arc complex. (For a discussion of arc and curve complexes see [Minsky 2000; 2001].)

Let $Z$ be a compact surface (possibly with boundary). Let $\mathscr{A}_{0}(Z)$ be the set of essential homotopy classes of simple closed curves or properly embedded arcs in $Z$. For simple closed curves homotopy class means the free homotopy class. For properly embedded arcs homotopy class means homotopy relative to the boundary of $Z$ if $Z$ is not an annulus or homotopy relative to endpoints if $Z$ is an annulus. The arc complex of $Z, \mathscr{A}(Z)$, is a simplicial complex whose simplices consist of $k+1$-tuples of distinct elements of $\mathscr{A}_{0}(Z)$ with pairwise disjoint representatives on $Z$. Making every simplex regular Euclidean of side length 1 gives $\mathscr{A}(Z)$ a path metric.

Let $S$ be a closed surface, $\mathscr{G} \mathscr{L}(S)$ be the geodesic lamination space of $S$ and $Y$ be an annular subsurface of $S$. Let $\hat{Y}$ be the unique compactified cover of $S$ determined by the inclusion of $\pi_{1}(Y)$ into $\pi_{1}(S)$. The boundary of $\hat{Y}$ is simply the quotient by $p i_{1}(Y)$ of the boundary at infinity of the universal cover minus the fixed points of a generator of $\pi_{1}(Y)$. Define a "projection map"

$$
\pi_{Y}: \mathscr{G} \mathscr{L}(S) \rightarrow \mathscr{A}(\hat{Y}) \cup \varnothing
$$

as follows: each $\lambda \in \mathscr{G} \mathscr{L}(S)$ lifts to $\hat{Y}$. The collection of all proper essential arcs of this lift determines a simplex of $\mathscr{A}(Y)$. We let $\pi_{Y}(\lambda)$ be the barycentre of this simplex. If there are no essential proper arcs in the lift of $\lambda, \pi_{Y}(\lambda)=\varnothing$.

Given an annular subsurface $Y$ of $S$ we define the $Y$-distance between laminations $\alpha, \beta$ in $\mathscr{G} \mathscr{L}(S)$ as $\mathrm{d}_{Y}(\alpha, \beta)=\mathrm{d}_{\mathscr{A}(Y)}\left(\pi_{Y}(\alpha), \pi_{Y}(\beta)\right)$.

Two notions of slenderness. A geometrically infinite end of a hyperbolic threemanifold is slender if it has an exiting sequence of geodesic pair-of-pants multicurves with length going to zero. To say that a sequence is exiting an end requires that all but finitely many of the geodesic pair-of-pants multicurves in the sequence are contained in any neighbourhood of the geometrically infinite end. We will call a hyperbolic 3-manifold slender if every geometrically infinite end of this manifold is slender.

Let $\lambda$ be an ending lamination associated to a geometrically infinite end $E$ of a hyperbolic 3-manifold without cusps, $N$. Let $X$ be the boundary component of a compact core of $N$ facing the end $E$, and set $g=$ genus $X$. Let $\alpha_{X}$ be a pair-ofpants multicurve on $X$. Let $\Phi$ be a collection of pairwise disjoint embedded annuli $A_{k}$ in $X(k=1, \ldots, 3 g-3)$ such that the collection of core curves of each annulus $A_{k}$ gives a pair-of-pants decomposition of $X$. Set

$$
\operatorname{diam}_{N}(\Phi)=\min _{A_{k} \in \Phi} d_{A\left(A_{k}\right)}\left(\pi_{A_{k}}(\lambda), \pi_{A_{k}}\left(\alpha_{X}\right)\right) .
$$


We say that the ending lamination $\lambda$ of $N$ is slender if and there exists a sequence $\left\{\Phi_{n}\right\}$ of collections of annuli whose core curves give pair-of-pants decompositions of $X$ (for each $n$ ) such that $\operatorname{diam}_{N}\left(\Phi_{n}\right) \rightarrow \infty$. Note that this definition does not depend upon the choice of multicurve $\alpha_{X}$ nor upon the choice of compact core defining $X$.

It is the deep results of Minsky [2000; 2001; 2003] that provide a link between these seemingly disparate notions of slenderness.

\section{The ending lamination conjecture}

We are now ready to prove the two theorems stated in the introduction. The proofs depend heavily on powerful technologies recently introduced to the field. The first is the theory of deformations of hyperbolic structures through hyperbolic conemanifolds developed by Hodgson and Kerckhoff [1998] and extended to geometrically finite hyperbolic 3-manifolds by Bromberg [2004]. The other technology is the theory of the complex of curves (and arcs) and projection coefficients developed by Minsky [2000; 2001].

For the remainder of this section we fix a closed orientable surface $S$ of genus at least 2. Given $P, Q \in \mathscr{D}(S)$ and markings $h_{P}: S \rightarrow P$ and $h_{Q}: S \rightarrow Q$, let $h: P \rightarrow Q$ be a homeomorphism in the homotopy class of the map $h_{Q} \circ\left(h_{P}\right)^{-1}$. We say that closed geodesics $p^{*} \subset P$ and $q^{*} \subset Q$ are the same (with respect to $\mathscr{D}(S))$ if $h\left(p^{*}\right)$ is in the same free homotopy class as $q^{*}$ and extend this definition to collections of geodesics in the obvious way.

Theorem 3.1. Suppose $P, Q \in \mathscr{D}(S)$ are slender singly degenerate hyperbolic 3manifolds without cusps with the same conformal boundary end-invariants and the same (w.r.t $\mathscr{D}(S))$ sequence of geodesic pair-of-pants multicurves with length going to zero. Then $P$ is isometric to $Q$. In fact, $P$ and $Q$ are equal as elements of $\mathscr{D}(S)$.

Proof. Let $\left\{p_{n}^{*}\right\}$ and $\left\{q_{n}^{*}\right\}$ be the respective sequences of geodesic pair-of-pants multicurves with length going to zero.

Let $X$ be the conformal boundary component of $P$ and $Q$. By the density theorem in [Bromberg 2002], $P$ and $Q$ lie in the boundary of the Bers slice $B_{X}$. So we may approximate $P$ and $Q$ (in the algebraic topology on $\mathscr{D}(S)$ ) by sequences of quasifuchsian manifolds within this Bers slice, say $\left\{P_{i}\right\}$ and $\left\{Q_{i}\right\}$, respectively. Call the conformal boundary component $X$ of $\left\{P_{i}\right\}$ and $\left\{Q_{i}\right\}$ indexing the Bers slice $B_{X}$ the bottom conformal boundary component.

For each $i$, let $\left\{\left(p_{n}^{i}\right)^{*}\right\}$ and $\left\{\left(q_{n}^{i}\right)^{*}\right\}$ be the corresponding sequences of geodesic pair-of-pants multicurves in $P_{i}$ and $Q_{i}$, respectively. Let $k_{m}$ be a sequence of positive numbers such that $\lim _{m \rightarrow 1} k_{m}=0$. We may pass to subsequences of $\left\{P_{i}\right\}$ and $\left\{Q_{i}\right\}$ relabelled as $\left\{P_{i}\right\}$ and $\left\{Q_{i}\right\}$ such that for each $i, \ell\left(\left(p_{i}^{i}\right)^{*}\right)<k_{i}$ and $\ell\left(\left(q_{i}^{i}\right)^{*}\right)<k_{i}$. 
Apply the drilling theorem [Bromberg 2004, Theorem 1.2] to the geodesic multicurves $\left(p_{i}^{i}\right)^{*}$ and $\left(q_{i}^{i}\right)^{*}$ in $P_{i}$ and $Q_{i}$, respectively, to get manifolds $\widetilde{P}_{i}$ and $\widetilde{Q}_{i}$, respectively. The multicurves $p_{i}^{i}$ and $q_{i}^{i}$ correspond to rank-2 cusps (zero coneangle) in $\widetilde{P}_{i}$ and $\widetilde{Q}_{i}$, respectively. Further, $\widetilde{P}_{i}$ and $\widetilde{Q}_{i}$ each have the same bottom conformal boundary component $X$ as $P_{i}$ and $Q_{i}$, respectively.

For each $i$, pass to a cover corresponding to this bottom conformal boundary component $X$ of $\widetilde{P}_{i}$ and $\widetilde{Q}_{i}$, respectively, to obtain new sequences $\left\{\bar{P}_{i}\right\}$ and $\left\{\bar{Q}_{i}\right\}$. See Figure 1.

The estimates provided by [Bromberg 2004, Theorem 8.2] (or Theorem 1.3 in the same reference) ensure that $\left\{\bar{P}_{i}\right\}$ and $\left\{\bar{Q}_{i}\right\}$ converge algebraically to $P$ and $Q$.

In $\bar{P}_{i}$ and $\bar{Q}_{i}$ the curves corresponding to the geodesic pair-of-pants multicurves $\left(p_{i}^{i}\right)^{*}$ and $\left(q_{i}^{i}\right)^{*}$ are now homotopic into rank-1 cusps. Thus $\bar{P}_{i}$ and $\bar{Q}_{i}$ are maximal cusps (in the sense of [McMullen 1991]). Further, each lies in the closure of the Bers slice $B_{X}$. Maximal cusps are unique in the boundary of a Bers slice (meaning they are uniquely determined by the corresponding pair-of-pants multicurve), so $\bar{P}_{i}$ is isometric to $\bar{Q}_{i}$ for each $i$. Hence $P$ and $Q$ are approximated by identical sequences in $\mathscr{D}(S)$ and are thus equal as elements of $\mathscr{D}(S)$.

Theorem 3.2. Suppose $P \in \mathscr{D}(S)$ is a singly degenerate hyperbolic 3-manifold without cusps with a slender ending lamination end-invariant and $Q \in \mathscr{D}(S)$ is a

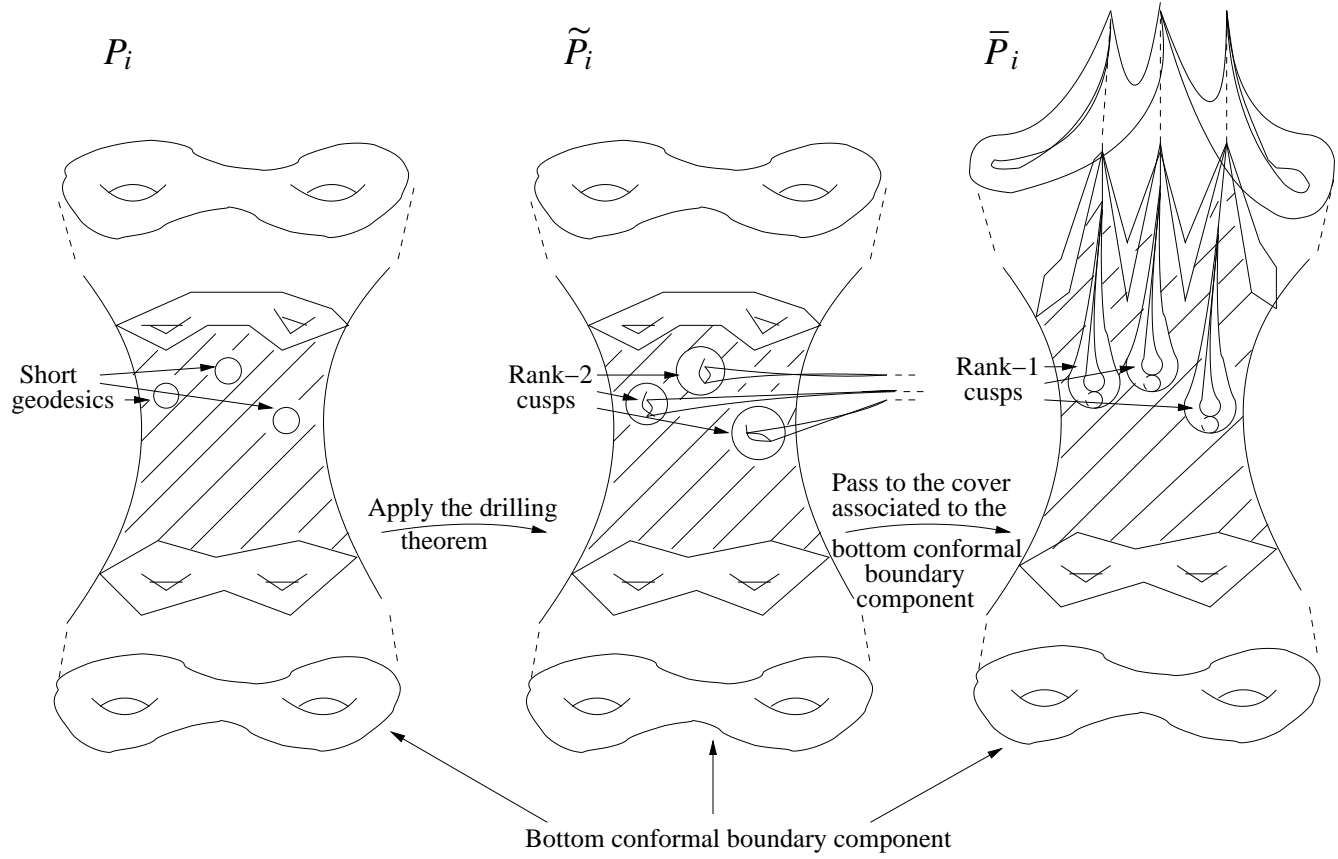

Figure 1. Producing the manifold $\bar{P}_{i}$ from the manifold $P_{i}$. 
hyperbolic 3-manifold with the same end-invariants as $P$. Then $P=Q$ in $\mathscr{D}(S)$; that is, there exists an isometry from $P$ to $Q$ in the homotopy class determined by markings of $P$ and $Q$ as elements of $\mathscr{D}(S)$.

Proof. Let $h: P \rightarrow Q$ be a homeomorphism respecting the markings of $P$ and $Q$ as elements of $\mathscr{D}(S)$. Fix a compact core $M_{P}$ of $P$. Then $M_{Q}=h\left(M_{P}\right)$ is a compact core of $Q$. Let $X_{P}$ be the boundary component of $M_{P}$ facing the geometrically infinite end of $P$ and let $X_{Q}=h\left(X_{P}\right)$. Since $P$ has a slender ending lamination end-invariant there is a sequence $\left\{\Phi_{n}\right\}$ of collections of annuli whose core curves give pair-of-pants decompositions of $X_{P}$ (for each $n$ ) such that $\operatorname{diam}_{P}\left(\Phi_{n}\right) \rightarrow \infty$. But then $\left\{h\left(\Phi_{n}\right)\right\}$ is a sequence of collections of annuli whose core curves give pairof-pants decompositions of $X_{Q}$ (for each $n$ ) such that $\operatorname{diam}_{Q}\left(h\left(\Phi_{n}\right)\right) \rightarrow \infty$. As the end-invariants of $P$ and $Q$ are the same, this implies that the ending lamination end-invariant of $Q$ is also slender.

Let $p_{n}^{*}$ and $q_{n}^{*}$ be the geodesic multicurves in $P$ and $Q$ representing the core curves of the annuli $\Phi_{n}$ and $h\left(\Phi_{n}\right)$, respectively. If $\lim _{n \rightarrow \infty} \operatorname{diam}_{P}\left(\Phi_{n}\right)=\infty$ and $\lim _{n \rightarrow \infty} \operatorname{diam}_{Q}\left(h\left(\Phi_{n}\right)\right)=\infty$, we know from [Minsky 2000, Theorem B] and the Bounded Geometry Theorem of [Minsky 2001] that $\left\{\ell\left(p_{n}^{*}\right)\right\}$ and $\left\{\ell\left(q_{n}^{*}\right)\right\}$ converge to zero; in other words, a slender ending lamination implies a slender end. The result now follows by Theorem 3.1.

\section{Examples: Singly degenerate manifolds without cusps with a slender ending lamination end-invariant.}

We now give a construction that demonstrates that singly degenerate hyperbolic 3-manifolds without cusps but with slender ending lamination end-invariants are abundant. Ours is a slight modification of an example of Minsky. See [Minsky 2000] for his construction and for a complete description of the techniques involved.

Fix a closed surface $S$ of genus at least 2 and a complete hyperbolic structure on $S$. Let $X$ be the conformal structure determined by this hyperbolic structure. We will construct a singly degenerate hyperbolic 3-manifold without cusps in the boundary of the Bers slice $B_{X}$ with a slender ending lamination end-invariant.

Let $\sigma=\left(\sigma_{1}, \ldots, \sigma_{n}\right)$ be a collection of simple closed curves of $X$ giving a pair-of-pants decomposition of $S$. Let $\alpha=\left(\alpha_{1}, \ldots, \alpha_{n}\right)$ and $\beta=\left(\beta_{1}, \ldots, \beta_{n}\right)$ be collections of simple closed curves giving pair-of-pants decompositions of the surface $S$ such that for each pair of integers $(i, j)$ with $1 \leq i, j \leq n$, the pairs $\left(\alpha_{i}, \beta_{j}\right),\left(\alpha_{i}, \sigma_{j}\right)$ and $\left(\beta_{i}, \sigma_{j}\right)$ bind the surface $S$.

Let $A_{1}, \ldots, A_{n}$ be disjoint embedded annuli in $\operatorname{int}(S)$ such that the core curve of $A_{j}$ is $\alpha_{j}, 1 \leq j \leq n$. Similarly define $B_{1}, \ldots, B_{n}$ using the curves $\beta_{1}, \ldots, \beta_{n}$. For each integer $i>0$, let $Y_{i}^{j}$ be the annulus $A_{j}$ if $i$ is odd, or $B_{j}$ if $i$ is even, 
$1 \leq j \leq n$. Let $\Phi_{i}$ be the homeomorphism of $S$ determined by doing a single Dehn-twist around the core-curve of each of the annuli $Y_{i}^{j}, 1 \leq j \leq n$ (the order in which the Dehn-twists are done being irrelevant since they commute).

Let $m_{i}$ be an increasing sequence of distinct positive integers. We now define homeomorphisms of the surface $S$ for each pair of positive integers. For each pair of positive integers $(p, q)$ with $p<q$ let $h_{p}^{q}=\Phi_{p}^{m_{p}} \circ \cdots \circ \Phi_{q-1}^{m_{q-1}}$. For a pair of positive integers $(q, p)$ with $q>p$ let $h_{q}^{p}=\left(h_{p}^{q}\right)^{-1}$. For pairs of positive integers $(p, p)$ let $h_{p}^{p}=$ identity. Observe that $h_{p}^{q} \circ h_{q}^{r}=h_{p}^{r}$.

For each conformal structure $X^{\prime}$ on $\operatorname{int}(S)$ there is a unique point in the Bers slice $B_{X}$ with conformal boundary $X \cup X^{\prime}$. We will consider the sequence of manifolds $N_{k}$ determined by the conformal structures $X$ and $h_{1}^{k}(X)$ for $k>0$. By compactness of the closure of the Bers slice $B_{X}$ we may pass to a subsequence such that there is a limit hyperbolic 3-manifold $N$.

For $1 \leq j \leq n$ and for positive $l$ let $Z_{l}^{j}=h_{1}^{l}\left(Y_{l}^{j}\right)$. By applying $h_{l}^{1}$ we obtain

$$
c_{l}^{j}(k)=d_{Y_{l}^{j}}\left(h_{l}^{1}(\sigma), h_{l}^{k}(\sigma)\right)
$$

. By making $m_{l}$ larger we can increase $c_{l}^{j}(k)$ independently of $k$ for each $j$. For each $j$, after possibly passing to a subsequence, let $c_{l}^{j}=\lim _{k \rightarrow \infty} c_{l}^{j}(k)$. Then we can ensure that $c_{l}^{j} \rightarrow \infty$ as $l \rightarrow \infty$ for each $j$.

Consider a limit manifold $N$. This has conformal end-invariant $X$ and ending lamination $\lambda=\lim _{k \rightarrow \infty} h_{1}^{k}(\sigma)$. The distances $d_{Z_{l}^{j}}(\sigma, \lambda)=c_{l}^{j}$. Thus $N$ has a slender end-invariant.

\section{Acknowledgments}

I thank Jeff Brock and Ken Bromberg for their encouragement and help in the development of this paper.

\section{References}

[Anderson 1998] J. W. Anderson, “A brief survey of the deformation theory of Kleinian groups", pp. 23-49 in The Epstein birthday schrift, edited by I. Rivin et al., Geom. Topol. Monogr. 1, Geom. Topol. Publ., Coventry, 1998. MR 99m:30086 Zbl 0904.30024

[Bonahon 1986] F. Bonahon, "Bouts des variétés hyperboliques de dimension 3", Ann. of Math. (2) 124:1 (1986), 71-158. MR 88c:57013 Zbl 0671.57008

[Brock et al. 2004] J. Brock, R. D. Canary, and Y. Minsky, "Classification of Kleinian surface groups, II: The ending lamination conjecture”, preprint, 2004. math.GT/0412006

[Bromberg 2002] K. Bromberg, "Projective structures with degenerate holonomy and the Bers density conjecture", preprint, 2002. math.GT/0211402

[Bromberg 2004] K. Bromberg, "Hyperbolic cone-manifolds, short geodesics, and Schwarzian derivatives”, J. Amer. Math. Soc. 17:4 (2004), 783-826. MR 2005h:57024 Zbl 1061.30037 
[Canary 1993] R. D. Canary, "Ends of hyperbolic 3-manifolds", J. Amer. Math. Soc. 6:1 (1993), 1-35. MR 93e:57019 Zbl 0810.57006

[Hodgson and Kerckhoff 1998] C. D. Hodgson and S. P. Kerckhoff, "Rigidity of hyperbolic conemanifolds and hyperbolic Dehn surgery”, J. Differential Geom. 48:1 (1998), 1-59. MR 99b:57030 Zbl 0919.57009

[Masur and Minsky 1999] H. A. Masur and Y. N. Minsky, "Geometry of the complex of curves, I: hyperbolicity", Invent. Math. 138:1 (1999), 103-149. MR 2000i:57027 Zbl 0941.32012

[Masur and Minsky 2000] H. A. Masur and Y. N. Minsky, "Geometry of the complex of curves, II: hierarchical structure", Geom. Funct. Anal. 10 (2000), 902-974. MR 2001k:57020 Zbl 0972.32011

[McMullen 1991] C. McMullen, "Cusps are dense", Ann. of Math. (2) 133:1 (1991), 217-247. MR 91m:30058 Zbl 0718.30033

[Minsky 1994] Y. N. Minsky, "On rigidity, limit sets, and end invariants of hyperbolic 3-manifolds", J. Amer. Math. Soc. 7:3 (1994), 539-588. MR 94m:57029 Zbl 0808.30027

[Minsky 1999] Y. N. Minsky, "The classification of punctured-torus groups", Ann. of Math. (2) 149:2 (1999), 559-626. MR 2000f:30028 Zbl 0939.30034

[Minsky 2000] Y. N. Minsky, "Kleinian groups and the complex of curves”, Geom. Topol. 4 (2000), 117-148. MR 2182094 Zbl 0953.30027

[Minsky 2001] Y. N. Minsky, "Bounded geometry for Kleinian groups", Invent. Math. 146:1 (2001), 143-192. MR 2004f:30032 Zbl 1061.37026

[Minsky 2003] Y. N. Minsky, "Classification of Kleinian surface groups, I: Models and bounds", preprint, 2003, Available at www.arXiv.org/abs/math.GT/0302208.

[Thurston 1979] W. P. Thurston, “The geometry and topology of three-manifolds", lecture notes, Princeton University, 1979, Available at http://msri.org/publications/books/gt3m.

Received February 21, 2006.

\section{RICHARD ALLEN EVANS}

revans@math.auckland.ac.nz

DEPARTMENT OF MATHEMATICS

UNIVERSITY OF AUCKLAND

PRIVATE BAG 92019

AUCKLAND

NeW ZEALAND 